ANNALES

POLONICI MATHEMATICI

IX (1961)

\title{
Über die Vektorkomitanten der Vektorfelder
}

von M. KUCHARZEWSKI (Katowice)

Einleitung. Es seien $m$ kontravariante Vektorfelder

(1)

$$
{ }_{1} u,{ }_{2} u, \ldots,{ }_{m} u
$$

mit den Koordinaten

(2)

$$
{ }_{1} u^{v},{ }_{2} u^{v}, \ldots,{ }_{m} u^{v}, \quad v=1,2, \ldots, n,
$$

im $n$-dimensionalen Raume $X_{n}$ gebegen.

Definition 1. Eine Vektorkomitante der Vektoren (1) nennen wir jedes Funktionensystem

$$
f^{\omega}\left({ }_{1} u^{v_{1}}, \ldots,{ }_{m} u^{\eta^{m}}\right), \quad \omega, v_{1}, \ldots, v_{m}=1,2, \ldots, n,
$$

der Koordinaten (2) dieser Vektoren, welches bei der beliebigen Transformation des Koordinatensystems

$$
x^{y^{\prime}}=x^{y^{\prime}}\left(x^{y}\right)\left({ }^{1}\right), \quad v^{\prime}=1^{\prime}, 2^{\prime}, \ldots, n^{\prime}
$$

der Beziehung

$$
f^{\omega^{\prime}}\left(A_{v}^{\nu_{1}^{\prime}} \cdot{ }_{1} u^{\nu}, \ldots, A_{v}^{\nu^{\prime} m} \cdot{ }_{m} u^{\nu}\right)=A_{\omega}^{\omega^{\prime}} f^{\omega}\left({ }_{1} u^{\nu_{1}^{\prime}}, \ldots,{ }_{m} u^{\nu^{\prime} m}\right)
$$

genügt, wo $A_{v}^{v^{\prime}}$ die Ableitungen

$$
A_{v}^{v^{\prime}}=\frac{\partial x^{v^{\prime}}}{\partial x^{v}}
$$

der Transformation (4) bedeuten.

Die Beziehung (5) kann auch in der Matrizenform geschrieben werden:

(6)

$$
f\left(A \cdot{ }_{1} u, \ldots, A \cdot{ }_{m} u\right)=A \cdot f\left({ }_{1} u, \ldots,{ }_{m} u\right) .
$$

Dann ist $A$ die Matrix

$$
A=\left\|A_{, \nu^{\prime}}\right\|
$$

(1) In dieser Arbeit bedienen wir uns der in [2] eingeführten Bezeichnungen (vergl. [2], Fußnote $\left(^{(1)}\right.$ und $\left({ }^{2}\right)$ ). 
und $f,{ }_{1} u, \ldots,{ }_{m} u$ stellen die einspaltigen Matrizen

$$
f=\left\|\begin{array}{c}
f^{1} \\
f^{2} \\
\vdots \\
f^{n}
\end{array}\right\|, \quad{ }_{\mu} u=\left\|\begin{array}{c}
{ }_{\mu} u^{1} \\
{ }_{\mu} u^{2} \\
\vdots \\
{ }_{\mu} u^{n}
\end{array}\right\|, \quad \mu=1,2, \ldots, m,
$$

dar. Endlich $A \cdot{ }_{\mu} u, A \cdot f$ bedeuten die Produkte der Matrizen (7) und (8). Ähnlich wie in der Arbeit [2] (Formel (9)) schreiben wir die Argumente der Funktion $f$ (die jetzt eine einspaltige Matrix darstellt) in der Form einer Matrix. Die $\mu$-te Spalte dieser enthält die Koordinaten des $\mu$-ten Vektors ${ }_{\mu} u$.

Die Gleichung (5) soll für beliebige .

$$
{ }_{\mu} u^{\nu}, \quad \nu=1,2, \ldots, n, \quad \mu=1,2, \ldots, m,
$$

und diejenige $A_{\nu}^{p^{\prime}}$ gelten, die der Ungleichung

$$
\text { (9) } \quad \operatorname{Det}\left\|A_{\nu}^{v^{\prime}}\right\| \neq 0
$$

genügen.

Die Gleichung (6) kann man auch in folgender für weitere Überlegungen bequemerer Form

$$
\bar{A} f\left(A \cdot{ }_{1} u, \ldots, A \cdot{ }_{m} u\right)=f\left({ }_{1} u, \ldots,{ }_{m} u\right)
$$

umschreiben. $\bar{A}$ bedeutet die zu $A$ inverse Matrix.

In dieser Arbeit bestimme ich alle Lösungen der Gleichung (10) und auf diese Weise erhalten wir alle Vektorkomitanten der $m$-kontravarianten Vektorfelder (1) im $n$-dimensionalen Raume.

Für den speziellen Fall $m=3$ und $n=2$ wurde dieses Problem von S. Gołąb [1] bei dor Gelegenheit der Untersuchungen über die Differentialvektorkomitanten erster Ordnung gelöst. Ein Teilergebnis in dieser Richtung ( $m=1$ und $n$ beliebig) hat A. Moór [3] erhalten als eine Folge aus dem allgemeineren Satze über Affinorkomitanten eines Vektorfeldes (S. 16, Satz 1, S. 17, Korollarium).

§ 1. Formulierung der Sätze. Wir betrachten ein bestimmtés System von Vektoren (1). Es seien $p$ von ihnen linear unabhängig. Ohne Beschränkung der Allgemeinheit kann man annehmen, daß eben die $p$ ersten von den Vektoren (1)

(11)

$$
{ }_{1} u, \ldots,{ }_{p} u
$$

linear unabhängig sind und jeder der Vektoren

$$
{ }_{p+1} u, \ldots,{ }_{m} u
$$

von (11) linear abhängig ist. Die eindeutig bestimmte Zahl $p$ erfüllt die Ungleichungen
(13)

Ist

so gibt es Zahlen

$$
p<m
$$

welche die Beziehungen

$$
e^{\lambda^{\alpha}}, \quad a=1,2, \ldots, p, \quad \varrho=p+1, \ldots, m,
$$

erfüllen. Die Skalaren (15) bleiben bei der affinen Transformation

$$
u^{\nu^{\prime}}=A_{\alpha}^{v^{\prime \prime}} u^{\nu}
$$

ungeändert. Da die lineare Abhängigkeit der Vektoren und die Zahl $p$ auch bei diesen Transformationen invariant sind, kann man die Form der Funktion $f$ für jeden Wert von $p$ unabhängig bestimmen. Dies ist in folgenden Sätzen enthalten.

SATz 1. Ist

$$
1 \leqslant p<m
$$

so hat jede Vektorkomitante (3) der Vektoren (1) folgende Gestalt:

(18) $f^{\omega}\left({ }_{1} u, \ldots,{ }_{m} u\right)={ }_{\beta} u^{\omega} \varphi^{\beta}\left({ }_{e} \lambda^{\alpha}\right), \quad \alpha, \beta=1, \ldots, p$,

$$
\omega=1,2, \ldots, n, \quad \varrho=p+1, \ldots, m,
$$

wo $\left.\varphi^{\beta}{ }_{(}{ }_{0} \lambda^{\alpha}\right)$ beliebige Funktion der Skalaren (15) bedeutet.

Die Sonderfälle $p=m$ und $p=0$ sind in den nächststehenden Sätzen erfaßt:

SATz 2. Ist

$$
p=m,
$$

so hat jede Vektorkomitante der Vektoren (1) die Gestalt

(19) $f^{\omega}\left({ }_{1} u, \ldots,{ }_{m} u\right)=C^{\alpha}{ }_{\alpha} u^{\omega}, \quad \alpha=1,2, \ldots, p, \quad \omega=1,2, \ldots, n$,

wo $C^{a}$ beliebige Konstanten bedeuten.

SATz 3. Sind alle Vektoren (1) gleich Null, so müssen die Komponenten der Vektorkomitante auch gleich $N$ ull sein.

Auf Grund der in der Arbeit [2] angeführten Überlegungen (Bẻmerkung 1 und 2), die auch jetzt gïltig sind, kann man annehmen, daß die aus $p$ ersten Reihen der Matrix

$$
\left\|{ }_{1} u, \ldots, p u\right\|\left({ }^{2}\right)
$$

gebildete Determinante von Null vèrschieden ist:

$$
\Delta=\left[{ }_{1} u, \ldots,{ }_{p} u\right] \neq 0 \text {. }
$$

(2) Die Symbole $\left\|_{1} u, \ldots, p_{p} u\right\|,\left[{ }_{1} u, \ldots,{ }_{2} u\right], \Delta_{\alpha / e}$ sind in der oben erwähnten Arbeit [2] (Bezeichnungen 1, 2, Bemerkung 3), erklärt, 
Außerdem gelten die Relationen

$$
{ }_{\varrho} \lambda^{\alpha}=\frac{\Delta_{\alpha / e}}{\Delta}, \quad \alpha=1,2, \ldots, p, \quad \varrho=p+1, \ldots, m .
$$

Aus dem Satze 1 folgt für $m=3$ und $n=2$ das Ergebnis, das S. Gołąb in seiner Arbeit [1] erhalten hat:

$$
f^{\omega}\left({ }_{1} u,{ }_{2} u,{ }_{3} u\right)={ }_{a} u^{\omega} \varphi^{\alpha}\left(\frac{\left[{ }_{3} u,{ }_{2} u\right]}{\left[{ }_{1} u,{ }_{2} u\right]}, \frac{\left[{ }_{1} u,{ }_{3} u\right]}{\left[{ }_{1} u,{ }_{2} u\right]}\right) .
$$

Diese Beziehung ist nur für $p=2$ und unter der Voraussetzung richtig, daß die zwei ersten der Vektoren ${ }_{1} u,{ }_{2} u,{ }_{3} u$ linear unabhängig sind. Ist dagegen $p=1$, d. h. gibt es nur einen zwischen ${ }_{1} u,{ }_{2} u,{ }_{3} u$ lịear unabhängigen Vektor, z. B.

$$
{ }_{1} u \neq 0,
$$

so ist die Funktion $f^{\omega}$ in der Form

$$
f^{\omega}\left({ }_{1} u,{ }_{2} u,{ }_{3} u\right)={ }_{1} u^{\omega} \varphi(\lambda, \mu)
$$

darstellbar, wo $\lambda$ und $\mu$ die Beziehungen

(23)

erfïllen. Ist z. B.

$$
{ }_{2} u=\lambda_{1} u, \quad{ }_{3} u=\mu_{1} u
$$

$$
{ }_{1} u^{1} \neq 0,
$$

so können wir $\lambda$ und $\mu$ aus (23) durch die Koordinaten der Vektoren ${ }_{1} u,{ }_{2} u,{ }_{3} u$ ausdrücken,

$$
\lambda=\frac{{ }_{2} u^{1}}{{ }_{1} u^{1}}, \quad \mu=\frac{{ }_{3} u^{1}}{{ }_{1} u^{1}},
$$

und die Formel (22) in der Form

$$
f^{\omega}\left({ }_{1} u,{ }_{2} u,{ }_{3} u\right)={ }_{1} u^{\omega} \varphi\left(\frac{{ }_{2} u^{1}}{{ }_{1} u^{1}}, \frac{{ }_{3} u^{1}}{{ }_{1} u^{1}}\right)
$$

darstellen. Ist endlich $p=0$, so müssen alle $f^{\omega}$ gleich Null sein.

Den Satz 2 kann man in diesem Falle nicht verwenden, weil die Gleichheit $p=m$ für $n=2$ und $m=3$ unmöglich ist.

Aus dem Satzen 2 folgt das Teilergebnis von A. Moór für $m=1$, welches alle Vektorkomitanten eines einzigen Vektors bestimmt. Dies besagt, daß jede Vektorkomitante des einzigen Vektors ${ }_{1} u$ zu diesem parallel ist:

$$
f^{\omega}\left({ }_{1} u\right)=C_{1} u^{\omega} \text {. }
$$

§ 2. Die Beweise der Sätze 1, 2 und 3. Zuerst machen wir noch eine Bemerkung, die in dem Beweise des Satzes 1 nützlich wird.
Bemerkung 1. Die Funktionen

$$
f^{\omega}\left({ }_{1} u^{{ }_{1}}, \ldots,{ }_{m} u^{{ }^{m} m}\right),
$$

die die Funktionalgleichung (5) erfüllen, müssen homogen erster Ordnung hinsichtlich der Veränderlichen

$$
{ }_{1} u^{\omega},{ }_{2} u^{\omega}, \ldots,{ }_{m} u^{\omega}
$$

und homogen nullter Ordnung hinsichtlich allen anderen Veränderlichen sein.

$$
{ }_{1} u^{\prime \prime},{ }_{2} u^{v}, \ldots,{ }_{m} u^{v}, \quad v \neq \omega,
$$

Diese Bemerkung folgt sofort aus der Beziehung ( $\left.{ }^{3}\right)$

$$
f^{\omega}\left(\varrho^{\nu_{1}}{ }_{1} u^{\nu_{1}}, \ldots, \varrho^{\nu_{m}}{ }_{m} u^{\nu_{m}}\right)=\varrho^{\omega} f^{\omega}\left({ }_{1} u^{\nu_{1}}, \ldots,{ }_{m} u^{\nu_{m}}\right),
$$

die man erhält, wenn in die Gleichung (5) als Elemente der Matrix $A$ die folgenden

eingesetzt werden.

$$
A_{v}^{\nu^{\prime}}=\varrho^{\nu^{\prime}} \delta_{v^{\prime \prime}}^{\nu^{\prime}}, \quad \varrho^{\nu^{\prime}} \neq 0,
$$

Beweis des Satzes 1. Wir nehmen an, daß die Zahl $p$ der Ungleichung

$$
1 \leqslant p<m
$$

genügt. Der Beweis wird durchgeführt, indem wir eine Matrix $A$ konstruiren, die die linke Seite der Gleichung (10) in die rechte von (18) überführt.

Wir unterscheiden zwei Fälle: $p<n$ und $p=n$. Es sei zuerst

$$
p<n \text {. }
$$

Da das Vektorensystem (1) und die Zahl $p$ die Bedingungen (20), (25) und (26) erfüllen, welche mit jenen in [2] auftretenden (20), (24) und (25) identisch sind, kann man ganz genau wie dort die Matrizen $B$ und $C$ bilden. Die Matrix $B$ hat folgende Gestalt:

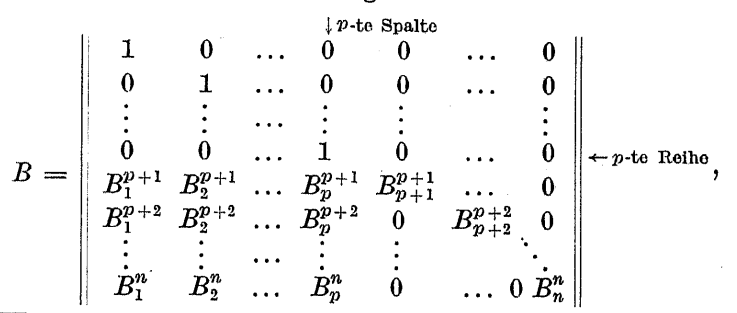

(3) In (24) haben wir die Akzenten über Indizes weggelassen. Da $f^{\omega^{\prime}}$ der Definition nach gleich $f^{\omega}$ sein muß und die mit dem Akzent versehene Indizes bei $\varrho$ und $\delta$ nur die Nummerierung der Argumente von $f^{\omega}$ bestimmen, führt dies zu keinen Mißverständnissen. 
wo die Elemente

(28) $B_{1}^{\sigma}, \ldots, B_{p}^{\sigma}, B_{\sigma}^{\alpha}, \quad \sigma=p+1, \ldots, n \quad$ (nicht summieren über $\sigma$ ), die Bedingungen

$$
\begin{gathered}
B_{\sigma}^{\sigma}=\Delta, \\
B_{1 a}^{\sigma} u^{1}+\ldots+B_{p a}^{\sigma} u^{p}+B_{\sigma a}^{\sigma} u^{\sigma}=0, \quad \alpha=1,2, \ldots, p,
\end{gathered}
$$

erfüllen. Die Matrix $C$ wählen wir wie folgt:

$$
C=\left\|\begin{array}{cccccc}
C_{1}^{1} & \ldots & C_{p}^{1} & 0 & \ldots & 0 \\
C_{1}^{2} & \ldots & C_{p}^{2} & 0 & \ldots & 0 \\
\vdots & & \vdots & \vdots & & \vdots \\
C_{1}^{p} & \ldots & C_{p}^{p} & 0 & \ldots & 0 \\
0 & \ldots & 0 & 1 & & \vdots \\
\vdots & & \vdots & & \ddots & 0 \\
0 & \ldots & 0 & \ldots & 0 & 1
\end{array}\right\|,
$$

wo $\left\|O_{\beta}^{\alpha}\right\|(\alpha, \beta=1,2, \ldots, p)$ eine zu $\left\|_{\beta} u^{a}\right\|$ inverse Matrix ist. Es gelten also die Beziehungen

$$
C_{\gamma \beta}^{a} u^{\gamma}=\delta_{\beta}^{\alpha}, \quad \alpha, \beta, \gamma=1,2, \ldots, p .
$$

Nachher bestimmen wir die Matrix $A$ als Produkt von $B$ und $C$ :

$$
A=C \cdot B \text {. }
$$

Die Matrix $A$ sieht falgendermaßen aus:

$$
A=\left\|\begin{array}{cccccc}
C_{1}^{1} & \ldots & C_{p}^{1} & 0 & \ldots & 0 \\
\vdots & & \vdots & \vdots & & \vdots \\
C_{1}^{p} & \ldots & C_{p}^{p} & 0 & \ldots & 0 \\
B_{1}^{p+1} & \ldots & B_{p}^{p+1} & B_{p+1}^{p+1} & & 0 \\
\vdots & \ldots & \vdots & \vdots & \ddots & \\
B_{1}^{n} & \ldots & B_{p}^{n} & 0 & \ldots & B_{n}^{n}
\end{array}\right\|
$$

Nun berechnen wir noch die in (10) auftretende, zu $A$ inverse Matrix $\bar{A}$. Gemäß der bekannten Formel kann man $\bar{A}$ als Produkt der zu $B$ und $C$ inversen Matrizen $\bar{B}$ und $\bar{C}$ erhaiten:
$(35)$

$$
\bar{A}=\bar{B} \cdot \bar{C} .
$$

Es ist leicht nachzuprüfen, daß $\bar{B}$ und $\bar{C}$ entsprechend folgende Formen besitzen:

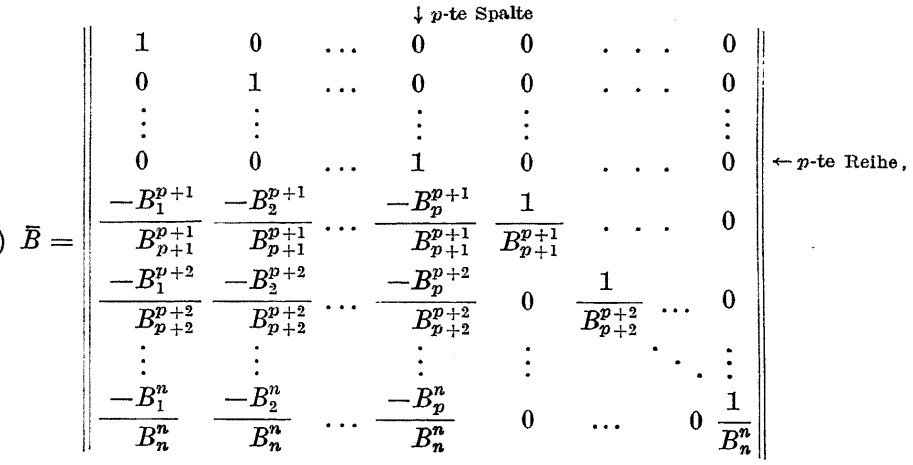$$
\bar{C}=\left\|\begin{array}{ccccccc}
{ }_{1} u^{1} & { }_{2} u^{1} & \ldots & { }_{p} u^{1} & 0 & \ldots & 0 \\
{ }_{1} u^{2} & { }_{2} u^{2} & \ldots & { }_{p} u^{2} & 0 & \ldots & 0 \\
\vdots & \vdots & & \vdots & \vdots & & \vdots \\
{ }_{1} u^{p} & { }_{2} u^{p} & \ldots & { }_{p} u^{p} & 0 & \ldots & 0 \\
0 & 0 & \ldots & 0 & 1 & \ldots & 0 \\
\vdots & \vdots & & \vdots & & \ddots & \vdots \\
0 & 0 & \ldots & 0 & 0 & \ldots & 1
\end{array}\right\| .
$$

Aus (35), (36) und (37) folgt mit Hilfe der Beziehungen (30), daß die Matrix $\bar{A}$ die Form

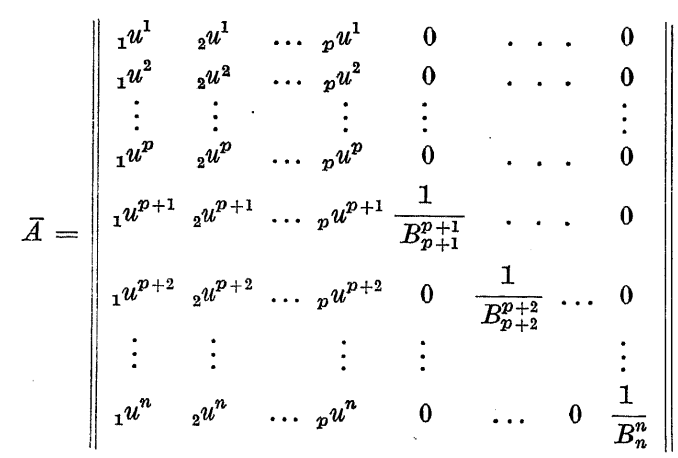

hat. 
Aus den in [1] ((45) und (47)) angeführten Formeln folgt, daß die in (10) auftretenden Produkte $A \cdot{ }_{\mu} u(\mu=1, \ldots, m)$ gleich
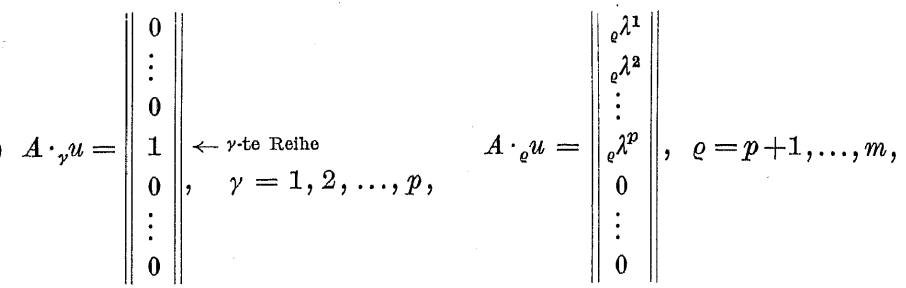

sind. Setzt man jetzt $A$ und $\bar{A}$ in die linke Seite von (10) ein, so erhalten wir folgende Gleichungen:

$(40)$

$$
\begin{array}{r}
f^{a}\left({ }_{1} u, \ldots,{ }_{m} u\right)={ }_{\beta} u^{\alpha} f^{\beta}\left(A \cdot{ }_{1} u, \ldots, A \cdot{ }_{m} u\right), \quad \alpha, \beta=1, \ldots, p, \\
f^{\sigma}\left({ }_{1} u, \ldots,{ }_{m} u\right)={ }_{\beta} u^{\sigma} f^{\beta}\left(A \cdot{ }_{1} u, \ldots, A \cdot{ }_{m} u\right)+\frac{1}{\Delta} f^{\sigma}\left(A \cdot{ }_{1} u, \ldots, A \cdot{ }_{m} u\right), \\
\sigma=p+1, \ldots, n .
\end{array}
$$

Jetzt ist es noch zu zeigen, daß

$$
f^{\sigma}\left(A \cdot{ }_{1} u, \ldots, A \cdot{ }_{m} u\right)=0, \quad \sigma=p+1, \ldots, n,
$$

falls $A$ die Form (34) hat.

Um dies zu zeigen, setzen wir in die zweite der Gleichungen (40) an Stelle ${ }_{1} u, \ldots,{ }_{m} u$ folgende, spezielle Werte:

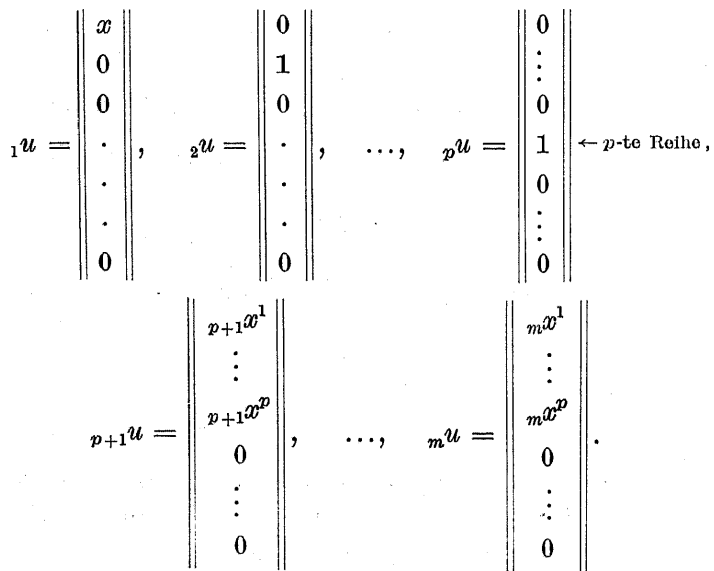

Dann erhalten wir mit Hilfe der Beziehungen (21):

(43)

$$
f^{\sigma}\left[\begin{array}{ccccccc}
x & 0 & \ldots & 0 & { }_{p+1} x^{1} & \ldots & { }_{m} x^{1} \\
0 & 1 & \ldots & 0 & p_{+1} x^{2} & \ldots & { }_{m} x^{2} \\
\vdots & & . & & \vdots & & \vdots \\
0 & 0 & & 1 & p_{p+1} x^{p} & \ldots & m^{x^{p}} \\
0 & 0 & \ldots & 0 & 0 & \ldots & 0 \\
\vdots & \vdots & & \vdots & \vdots & & \vdots \\
0 & 0 & \ldots & 0 & 0 & \ldots & 0
\end{array}\right]=\frac{1}{x} f^{\sigma}\left[\begin{array}{ccccccc}
1 & 0 & \ldots & 0 & { }_{p+1} x^{1} / x & \ldots & { }_{m} x^{1} / \bar{x} \\
0 & 1 & \ldots & 0 & p_{+1} x^{2} & \ldots & { }_{m} x^{2} \\
\vdots & \ddots & & & \vdots & & \vdots \\
0 & 0 & & 1 & p_{p+1} x^{p} & \ldots & { }_{m} x^{p} \\
0 & 0 & \ldots & 0 & 0 & \ldots & 0 \\
\vdots & \vdots & & \vdots & \vdots & & \vdots \\
0 & 0 & \ldots & 0 & 0 & \ldots & 0
\end{array}\right]
$$

Die Funktionen $f^{\sigma}(\sigma=p+1, \ldots, n)$ sind aber auf Grund der Bemerkung 1 homogen nullter Ordnung in Bezug auf

$$
{ }_{1} u^{1}, \ldots,{ }_{m} u^{1} \text {. }
$$

Aus (43) folgt also die Beziehung

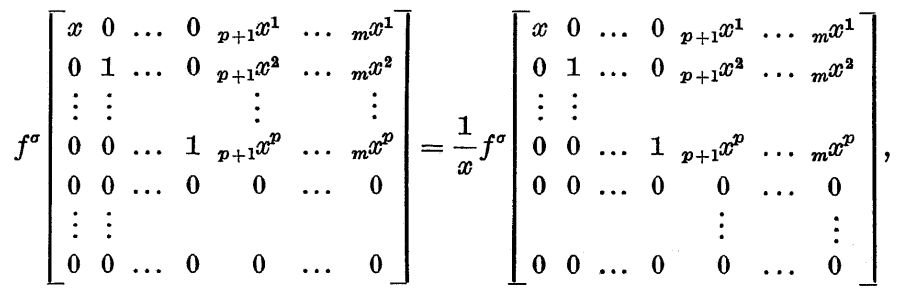

wenn man die erste Reihe der Matrix, welche in der rechten Seite von (43) steht, mit $x$ multipliziert.

Wird nun in dieser Gleichung $x=2$ gesetzt, so ergibt sich

$$
f^{\sigma}\left[\begin{array}{ccccccc}
2 & 0 & \ldots & 0 & p_{+1} x^{1} & \ldots & m^{x^{1}} \\
0 & 1 & \ldots & 0 & { }_{p+1} x^{2} & \ldots & m^{x^{2}} \\
\vdots & \vdots & & \vdots & \vdots & & \vdots \\
0 & 0 & \ldots & 1 & { }_{p+1} x^{p} & \ldots & { }_{m} x^{p} \\
0 & 0 & \ldots & 0 & 0 & \ldots & 0 \\
\vdots & \vdots & & \vdots & \vdots & & \vdots \\
0 & 0 & \ldots & 0 & 0 & \ldots & 0
\end{array}\right]=0
$$

Da $f^{\sigma}$ homogen nullter Ordnung hinsichtlich den in erster Reihe stehenden Veränderlichen sind und ${ }_{p+2} x^{1}, \ldots,{ }_{m} x^{1}$ ganz beliebig sind, folgt die Beziehung (41). Bezeichnen wir $\varphi^{\beta}\left({ }_{Q} \lambda^{\alpha}\right) \stackrel{d f}{=} f^{\beta}\left(A \cdot{ }_{1} u, \ldots, A \cdot{ }_{m} u\right) \quad(\alpha, \beta=$ 
$=1,2, \ldots, \varrho ; \varrho=p+1, \ldots, m)$, so ergibt sich aus (40) und (39), daß das Funktionensystem

$$
f^{\omega}\left({ }_{1} u, \ldots,{ }_{m} u\right), \quad \omega=1,2, \ldots, n,
$$

die durch (18) bestimmte Form haben muß. Auf diese Weise ist der Beweis des Satzes 1 im Falle $n<p$ beendet. Den Beweis dieses Satzes im Falle $n=p$ erhalten wir, wenn man in allen vorherstehenden Überlegungen an Stelle $B$ die Einheitsmatrix $\left\|\delta_{\omega}^{\prime}\right\|(\nu, \omega=1,2, \ldots, n)$ nimmt.

Der Beweis des Satzes 2. Jetzt nehmen wir an, daß es

gilt. Wird in (5)

$$
m=p \leqslant n
$$

$$
{ }_{a} u^{v}=\delta_{a}^{v}, \quad v=1,2, \ldots, n, \quad \alpha=1,2, \ldots, p=m,
$$

eingesetzt, so ergibt sich daraus

$$
f^{\omega}\left(A_{1}^{v_{1}}, A_{2}^{v_{2}}, \ldots, A_{m}^{y_{m}}\right)=A_{v}^{\omega^{\prime}} f^{\prime}\left(\delta_{1}^{\nu_{1}}, \delta_{2}^{\gamma_{2}}, \ldots, \delta_{m}^{\eta_{m}}\right) .
$$

Ist

$$
m=p=n,
$$

so folgt ohne weiteres die Beziehung (19), wenn man in (45) $A_{a}^{\gamma^{\prime \prime}}={ }_{a} u^{p}$ und $f^{y}\left(\delta_{1}^{y_{1}}, \ldots, \delta_{m}^{y_{m}}\right)=O^{y}$ einsetzt. Falls

$$
m=p<n
$$

ist, müssen wir zuerst zeigen, daß alle

$$
f^{\sigma}\left(\delta_{1}^{\nu_{1}}, \delta_{2}^{\nu_{2}}, \ldots, \delta_{m}^{\nu_{m}}\right), \quad \sigma=p+1, \ldots, n,
$$

gleich Null sind. Um dies zu beweisen setzen wir in (45)

$$
\begin{array}{ll}
A_{a}^{\nu_{1}}=\delta_{\alpha}^{y_{1}}, & \alpha=1,2, \ldots, p, \\
A_{\sigma}^{\nu_{1}}=-\delta_{\sigma}^{\nu_{1}}, & \sigma=p+1, \ldots, n,
\end{array}
$$

ein. Dann erhalten wir

$$
f^{\sigma}\left(\delta_{1}^{y_{1}}, \delta_{2}^{y_{2}}, \ldots, \delta_{m}^{\eta_{m}}\right)=-f^{\sigma}\left(\delta_{1}^{\nu_{1}}, \ldots, \delta_{m}^{y_{m}}\right), \quad \sigma=p+1, \ldots, n .
$$

Daraus folgt sofort (46) und der Beweis des Satzes ist beendet.

Der Beweis des Satzes 3. Um die Werte der Funktion

$$
f^{\omega}, \quad \omega=1,2, \ldots, n,
$$

zu bestimmen, falls alle Vektoren (1) gleich Null sind, setzt man

$$
A_{v}^{\omega^{\prime}}=-\delta_{v}^{\omega}, \quad \omega, v=1,2, \ldots, n,
$$

in (5) ein. Dann erhalten wir

$$
f^{\omega}\left(\begin{array}{cccc}
0 & 0 & \ldots & 0 \\
\vdots & \vdots & & \vdots \\
0 & 0 & \ldots & 0
\end{array}\right)=-f^{\omega}\left(\begin{array}{cccc}
0 & 0 & \ldots & 0 \\
\vdots & \vdots & & \vdots \\
0 & 0 & \ldots & 0
\end{array}\right), \quad \omega=1,2, \ldots, n .
$$

Daraus folgt, daß die den Nullvektoren entsprechenden Werte von $f^{\omega}$ gleich Null sein müssen.

\section{Literaturverzeichnis}

[1] S. Gołab, Über Differentialkomitanten der Vektorfelder (unter der Presse). [2] M. Kucharzewski, Über die skalaren Komitanten der Vektorfelder, Ann. Pol. Math. 9 (1961), S. $311-323$.

[3] A. Moór, Über Tensoren, die aus angegebenen geometrischen Objekten gebildet sind, Publ. Math. 6 (1959), S. 15-25.

Reģu par la Rédaction le 20. 3. 1960 